\title{
A generic framework for video understanding applied to group behavior recognition
}

\author{
Sofia Zaidenberg, Bernard Boulay, François Brémond \\ Inria \\ STARS team \\ 2004 Route des Lucioles - BP 93 \\ 06902 Sophia Antipolis (France) \\ firstname. namedinria.fr
}

\begin{abstract}
This paper presents an approach to detect and track groups of people in video-surveillance applications, and to automatically recognize their behavior. This method keeps track of individuals moving together by maintaining a spacial and temporal group coherence. First, people are individually detected and tracked. Second, their trajectories are analyzed over a temporal window and clustered using the Mean-Shift algorithm. A coherence value describes how well a set of people can be described as a group. Furthermore, we propose a formal event description language. The group events recognition approach is successfully validated on 4 camera views from 3 datasets: an airport, a subway, a shopping center corridor and an entrance hall.
\end{abstract}

\section{Introduction}

In the framework of a video understanding system (figure 11, video sequences are abstracted in physical objects: objects of interest for a given application. Then the physical objects are used to recognize events. In this paper, we are interested by the group behavior in public spaces. Given a set of detected and tracked people, our task is finding associations of those people into spatially and temporally coherent groups, and detecting events describing group behavior.

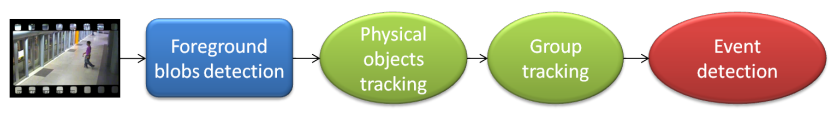

Figure 1. Description of the proposed video understanding system

Tracking people, and especially groups of people in relatively an unconstrained, cluttered environment is a challenging task for various reasons. In [8], Ge et al. propose a method to discover small groups of people in a crowd based on a bottom-up hierarchical clustering approach. Trajectories of pedestrians are clustered into groups based on their closeness in terms of distance and velocity. The experiments of this work have been made on videos taken from a very elevated viewpoint, providing few occlusions. Haritaoglu et al. [9] detect and track groups of people as they shop in a store. Their method is based on searching strongly connected components in a graph created from trajectories of individual people, following the idea that people belonging to the same group have a lower inter-distance. This method however does not allow group members to move away and return to the group without being disconnected from it. Furthermore, the application of a shopping queue lacks genericity (people are rather static and have a structured behavior), it is not clear how well this method is adaptable to another context of use. Other approaches, such as [14], aim at detecting specific group-related events (e.g. queues at vending machines) without tracking. Here again, the method does not aim at consistently tracking a group as its dynamics vary. In [10], an algorithm for group detection and classification as voluntary or involuntary (e.g. assembled randomly due to lack of space) is proposed. A top-down camera is used to track individuals, and Voronoi diagrams are used to quantify the sociological concept of personal space. No occlusion handling is done in this work hence the applicability to other points of view of the camera or to denser scenes is questionable. Figure 2 shows the result of our event recognition on tracked groups on a dataset recorded at the Eindhoven airport.

Event recognition is a key task in automatic understanding of video sequences. In this work we are mainly interested in group events, but the usual techniques can be applied to different kinds of objects (person, vehicle, group,...). The typical detection algorithm (figure 1) takes as input a video sequence and extracts interesting objects (physical objects). This abstraction stage is the layer be- 

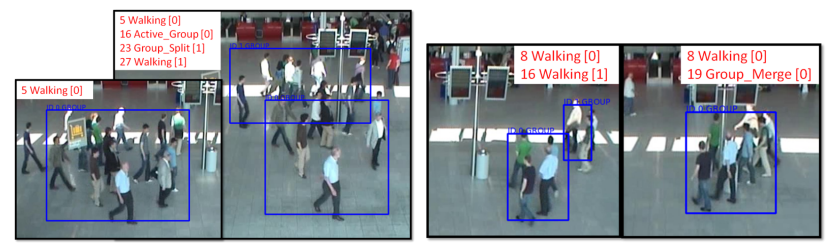

Figure 2. Group event recognition on Eindhoven airport sequences. Left: The group is detected as splitting into 2 sub-groups. Right: Two groups are detected as merging into one.

tween the image and the semantic worlds. Then, these objects of interest are used to model events. Finally, the events are recognized. The abstraction stage determines which modeling techniques can be applied.

The possible abstraction technique can be pixel based [1] or object based [17]. The first kind of techniques is not well adapted for groups. Indeed, persons belonging to the same group are not necessary physically linked. With object abstraction, a video sequence is represented thanks to the detected objects (persons, vehicles,...) and their associated properties (speed, trajectory,...). In the literature, several approaches use this abstraction level [16], because an activity can be naturally modeled based on those properties.

Lavee et al. [11] classify existing event modeling techniques in three categories: the pattern recognition models, the state based models and the semantic models.

The pattern recognition models are classical recognition techniques using classifiers as the nearest neighbor method, boost techniques, support vector machines and neural networks [4]. These techniques are well formalized. But adding new types of events implies the training of new classifiers.

The state based models formalize the events in spatial and temporal terms using semantic knowledge: Finite State Machines (FSM), Bayesian Networks (BN), Hidden Markov Models (HMM), Dynamic Bayesian Networks (DBN) and Conditional Random Fields (CRF). The HMMs and all their variants are heavily used for event modeling [6]. They take the advantages of the FSM (temporal modeling) and of the BNs (probabilistic modeling). But due to the nature of the HMMs (time sliced structure), the complex temporal relations (e.g. during) are not easily modeled. Lin et al. [12] propose an asynchronous HMM to recognize group events. Brdiczka et al. construct in [2] HMMs upon conversational hypotheses to model group events during a meeting. One drawback of the modified HMM methods is that since the classical structure of HMMs is modified, efficient algorithms can not be applied without approximation.

The semantic models define spatio-temporal relations between sub-events to model complex events. Due to the nature of these models, the events must be defined by an expert of the application domain. Moreover, these models are often deterministic. Several techniques are studied: grammar based models, Petri nets (PN), constraint solving models and logic based models.

As shown in this section, the quantity of techniques for abstraction and event modeling is huge. In this paper, we propose a framework (ScReK: Scenario Recognition based on Knowledge) to easily model the semantic knowledge of the considered application domains: the objects of interest and the scenario models, and to recognize events associated to the detected group based on spatio-temporal constraints.

In the rest of this paper, we first describe our technique to detect and track groups of people (section 2), then we describe our event detection method applied to tracked groups (section 3). Section 4 presents evaluations.

\section{Group Tracking}

Given a set of detected and tracked people, the proposed method focuses mainly on the task of finding associations of those people into spatially and temporally coherent groups. The human definition of a group is people that know each other or interact with each other. In fact, according to McPhail [13]: Two defining criteria of a group [are] proximity and/or conversation between two or more persons. It is quite difficult to directly detect people interactions and conversation in a video or the fact that people know each other. For automatic recognition we derive this definition: two or more people who are spatially and temporally close to each other and have similar direction and speed of movement, or better: people having similar trajectories.

Group tracking is based on people detection. The people detection can be performed by various methods. We have compared several methods and chosen the best one, it is based on background-subtraction described in [18] because of the quality of its results (see table 1 for a comparison of several methods).

Blobs of foreground pixels are grouped to form physical objects (also called mobiles) classified into predefined categories based on the 3D size of objects (using a calibrated camera): GROUP_OF_PERSONS, PERSON and NOISE. When people overlap (which happens quite often with a low viewpoint, such as in figure 6) or are too close to each other, segmentation fails to split them and they are detected as a single object classified as GROUP_OF_PERSONS because its size is bigger than the size of a single person. Thoses classes of objects are specified using gaussian functions. Mean, sigma, min and max values are provided for each class and a score is computed representing how well an object's dimensions fit in each category. The category with the best score is assigned as the class of the object. Detected objects at each frame are tracked consistently on the long term using a multiple feature-based tracker [3].

Individual trajectories are the input of the group tracking algorithm, which is divided into four parts: creation, update, 


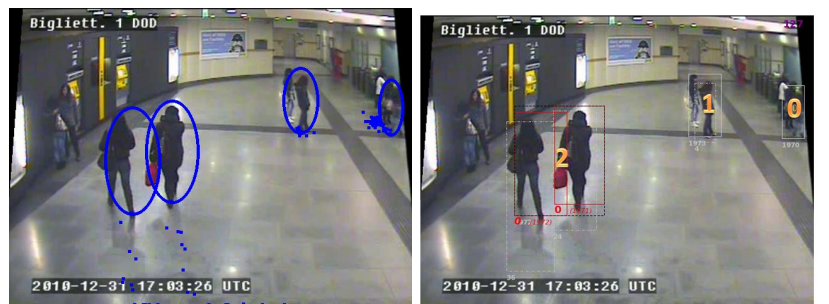

Figure 3. Example of 5 tracked people clustered into 3 trajectory clusters. A group is created from cluster 2 .

split/merge and termination. In order to detect temporally coherent groups, we observe people trajectories over a time window, denoted delay $T$. In the experiments presented section 4, we used $T=20$ frames. Working at frame $t_{c}-T, t_{c}$ being the current frame of the video stream, we cluster trajectories of individuals between frames $t_{c}-T$ and $t_{c}$ to find similar trajectories, representative of groups. We choose the Mean-Shift clustering algorithm [7] because it does not require to set as input the number of clusters. However, Mean-Shift does require a tolerance parameter determining the size of the neighborhood for creating clusters. Figure 3 shows the input prints and the clustering result.

A trajectory is defined as Traj $=\left\{\left(x_{i}, y_{i}\right), i=\right.$ $0 \ldots T-1\} \cup\left\{\left(s_{x_{i}}, s_{y_{i}}\right), i=1 \ldots T-1\right\}$ where $\left(x_{i}, y_{i}\right), i \in$ $[0 ; T-1]$ in each trajectory is the position of a group in the same frame $i$, and $\left(s_{x_{i}}, s_{y_{i}}\right)=\operatorname{speed}(i-1, i), i \in[1 ; T-1]$ is the speed of the group between frames $i-1$ and $i$. If $k$ positions on the trajectory are missing because of lacking detections, we interpolate the $k$ missing positions between known ones. Each trajectory is a point in a $2(2 T-1)$ dimensional space. Mean-Shift is applied on a set of such points. To make the approach more generic and being able to add other features, we normalize the values using minimum and maximum ranges. The range of positions on the ground plane is determined by the field of view. The minimum speed is 0 and the maximum speed is set to $10 \mathrm{~m} / \mathrm{s}$, greatly exceeding all observed values. From the raw value of $x, y$ and $s$ (the speed) denoted by $r \in[$ min, max $]$, we compute the corresponding normalized value $n \in[0,1]$ as: $n=\frac{r-\min }{\max -\min }$, where $\min$ and $\max$ are the respective minimum and maximum values. We set the tolerance to 0.1 , considering grouping trajectories distant by less than $10 \%$ of the maximum. This value is quite low because clustering is used only to group very close people, the case where people temporarily split being handled by the update step described below.

We characterize a group by three properties: the average over the frames in which the group is detected of the inter-mobile distance and the average over frames of standard deviations of speed and direction. These properties enable the definition of a coherence criterion: groupIncoherence $=\omega_{1}$.distanceAvg + $\omega_{2} \cdot$ speedStdDev $+\omega_{3} \cdot$ directionStdDev, where the weights $\omega_{1}, \omega_{2}$ and $\omega_{3}$ are normalization parameters. We use $\omega_{1}=7$ and $\omega_{2}=\omega_{3}=5$ to slightly favor distance over speed and direction similarity which are quite noisy. With this definition, a low value of groupIncoherence is significative of a group.

Groups are created from clusters of more than one physical object. In the case where one GROUP_OF_PERSONS object is detected at frame $t_{c}-T$, we analyze its trajectory through the time window. If this object stays the size of a group, or is close to other objects, we can create a group and compute its groupIncoherence. If the resulting value is low enough, we keep the created group. In case of a single GROUP_OF_PERSONS object, the groupIncoherence value is naturally very low because of a null distanceAvg component. The creation step is made up of these two cases.

Group dynamics vary. Sometimes all group members do not have similar trajectories, for example when the group is waiting while one member buys a ticket at a vending machine. Clustering is not enough to correctly update an existing group in that case. First, we try to associate clusters with groups existing at the previous frame, using the notion of probable group of a mobile, defined hereafter. During tracking, mobiles detected at different frames are connected by probabilistic links in order to track consistently the same real objects. We use the term father and son for the mobiles resp. in the oldest and most recent frame of the link. If a father, within a window of $T$ frames, of the mobile $m$ was in a group $g$ and the link probability between father and son is above a given threshold (a value of 0.6 is usually used in the experiments section 4), then the father's group $g$ is called the probable group of the mobile $m: P G(m)=g$. Each cluster $c$ is associated with the probable group of most mobiles in the cluster: $G(c)=\operatorname{argmax}_{g \in\left\{g_{i}^{c}\right\}} \mid\left\{g_{i}^{c} \mid g_{i}^{c}=\right.$ $g\} \mid$, where $G(c)$ is the group associated with cluster $c$ and $\left\{g_{i}^{c}\right\}=\left\{P G\left(m_{i}^{c}\right)\right\}$ the set of probable groups of mobiles belonging to cluster $c\left(\left\{m_{i}^{c}\right\}\right.$ being the set of mobiles in cluster $c$ ). Several clusters can be associated to the same group, ensuring that group members having temporarily diverging trajectories will be kept in the group for a minimal amount of time. Each mobile $m_{i}^{c}$ is added to the group $G(c)$ if this group is really the probable group of the considered mobile: $P G\left(m_{i}^{c}\right)=G(c)$. In fact, the update step aims at tracking existing members of the group and not new comers. This procedure is summarized in algorithm 1

The split of groups operates naturally. When a mobile from a group has moved away for too many frames, its probable group becomes empty and it cannot be added to an existing group during the update step, so it splits. It may be part of a new group in the creation step, if it gets clustered together with other mobiles.

Two groups $g_{1}$ and $g_{2}$ can be merged if two mobiles, one in each group at frame $t_{c}-T+k(k \in[0 ; T-1])$, have the same son at frame $t_{c}-T+l(l \in[k+1 ; T-1])$, meaning that the two mobiles will merge. The oldest group among 


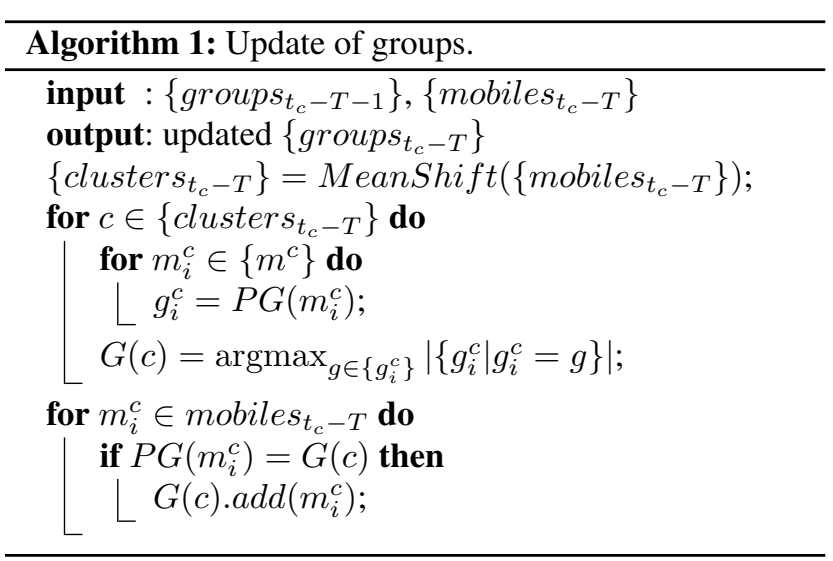

$g_{1}$ and $g_{2}$ is kept and all mobiles of the disappearing group are added into the remaining group.

The group termination step erases old groups. Mobiles that have been detected at a largely outdated frame (e.g. $\left.t_{c}-5 T\right)$ are deleted at frame $t_{c}-T$ and empty groups are erased. As a consequence, groups having no new mobiles for $5 T$ frames are erased. All existing groups, even currently empty ones, can potentially be updated.

Finally, the output of the group tracker, which is the input of the event detection, is a set of tracked groups (keeping a consistent id through frames) having properties (such as the intra-objects distance) and composed of detected physical objects at each frame.

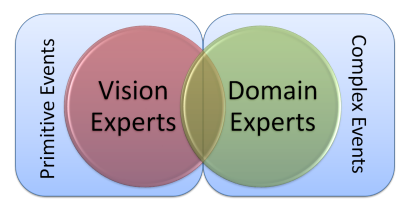

Figure 4. Knowledge modeling for video event recognition.

\section{Event Recognition: a Generic Framework}

In this work, a generic framework for event recognition is proposed $(S c R e K)$. The motivation of this framework is to use it within any video understanding application. The genericity is obtained in terms of objects of interest and event models. We can identify two main parts in an event recognition process: the application knowledge (what are the expected objects? what are the event models?) and the event recognition algorithm.

Knowledge representation is a key issue for genericity. We believe that the knowledge should be modeled with the collaboration of two different categories of people (figure 4): vision experts (specialists in vision algorithms), and application domain experts (specialists in the expected events of their domain). Vision experts are modeling the objects of interest (detected by vision algorithms) and the video primitives (properties computed on the detected objects of interest). Domain experts have to model the expected application events.

Usually, for video event recognition, knowledge is represented using OWL (Web Ontology Language). Even with tools like Protégé, it is difficult for a non computer specialist to create her/his own model without a long and tedious learning of the OWL formalism. The ScReK framework proposes its own declarative language to easily describe the application domain knowledge: the ontology. $\mathrm{ScReK}$ proposes a grammar description of the objects and events using the extended BNF (Backus Naur Form) representation. $O_{i}$, is described by its parent, $O_{j}$, and its attributes: $O_{i}=\left\{a_{k}\right\}_{k=0, \ldots O_{i}^{n}}$. The objects are defined using an inheritance mechanism. The object $O_{i}$ inherits all the attributes of its parent $O_{j}$. The attributes are described with the help of basic types. 11 basic types are predefined: boolean, integer, double, timestamp, time interval, 2D point (integer and double), 3D point (integer and double), and list of 3D points. The user can contribute by adding new basic types. Moreover, a history of the values of a basic type is automatically stored. It is useful for vision primitives based on the evolution of a value in time (e.g. trajectory).

For group behavior recognition, detected group objects within the video sequence and scene context objects (zone, equipment) are described. The scene context objects help to recognize specific events (e.g. by defining a forbidden access zone or a threshold). For instance, the class of group objects is defined as follows in the ScReK language:

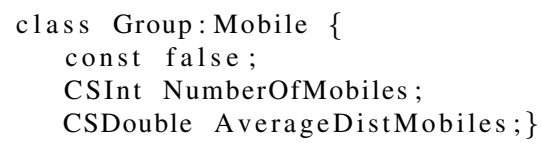

A Group is a Mobile and it inherits all the attributes of a Mobile object (3D size, 3D position,...). A Group is not constant (dynamic, i.e. its attributes values can change throughout time). One of its attributes, NumberOfMobiles is the number of objects which compose the group.

The second kind of knowledge to represent is the event models. They are composed of 6 parts: (1) the type of the scenario can be one of the following: PrimitiveState, CompositeState, PrimitiveEvent, CompositeEvent, from the simplest to the most complex events. (2) the name of the event model which can be referenced for more complex events. (3) the list of physical objects (i.e. objects of interest) involved in the event. The type of the objects is depending on the application domain. (4) the list of components contains the sub-events composing the event model. (5) the list of constraints for the physical objects or the components. The constraints can be temporal (between the components) or symbolic (for physical objects). (6) the alarm information describes the importance of the scenario model in terms of urgency. Three values are possible, from less urgent to more urgent: NOTURGENT, URGENT, VERYURGENT. 
The alarm level can be used to filter recognized events, for displaying only important events to the user. Hereafter is a sample event model:

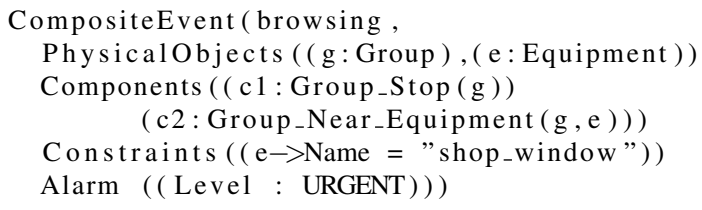

The application domain expert models the event browsing by "a group is stopped in front of the shop-window" with the model above. The vision expert models the subevent Group_Near_Equipment (by measuring the distance between a group and an equipment) and Group_Stop (by computing the speed of a group).

The last part of the event recognition framework is the recognition algorithm itself. The proposed algorithm solves spatio-temporal constraints on the detected groups. The usual algorithms to recognize such events can be time consuming. The $S c R e K$ framework proposes to define optimal event models: at most two components, at most one temporal constraint (Allen's algebra) between these components. This property is not restrictive since all event models can be optimized in this format. Thanks to the optimal property, the event model tree is computed. The tree defines which sub-event (component) triggers the recognition of which event: the sub-event which happens last in time triggers the recognition of the global event. For instance, the event A has two components B and $\mathrm{C}$ with constraint: B before $\mathrm{C}$. The recognition of $\mathrm{C}$ triggers the recognition of $\mathrm{A}$. The tree triggers the recognition of the only events that can happen, decreasing the computation time.

The first step of the event recognition process is to recognize all the possible simple events (most of these events are based on the vision primitives) by instantiating all the models with the detected objects (e.g. instantiating the model Group_Stays_Inside_zone (takes as input one group and one zone) for all the detected groups and all the zones of the context). The second step consists in recognizing complex events according to the event model tree and the simple events previously recognized. The final step checks if the recognized event at time $t$ has been already recognized previously to update the event (end time) or create a new one.

\section{Results}

People detection is an input to group detection. We compared several methods to validate our choice of method. Table 1 sums up the results of an evaluation done on a 36006 frames sequence (approximately 2 hours of video) in which 37 ground truth (GT) objects (people) have been annotated. [5] is a feature-based people detector whereas [15] and [18]

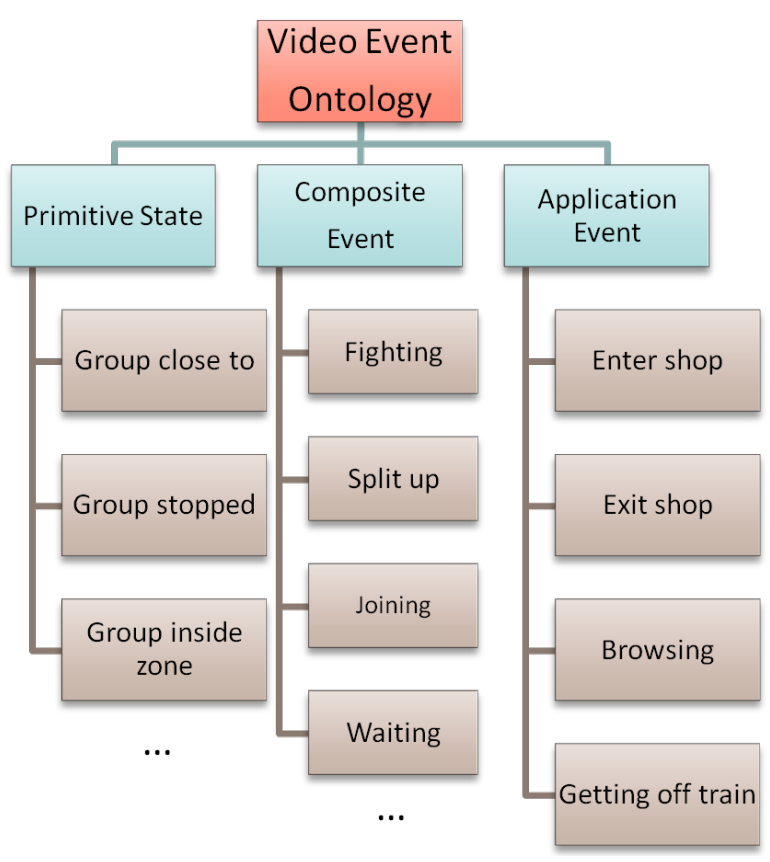

Figure 5. Proposed group event ontology.

\begin{tabular}{|c|c|c|c|c|}
\cline { 2 - 5 } \multicolumn{1}{c|}{} & {$[5]$} & {$[15]$} & $\mathrm{C}$ & {$[18]$} \\
\hline True Positives (TP) & 3699 & 3897 & 4547 & 6559 \\
\hline False Positives (FP) & 1379 & 185 & 125 & 128 \\
\hline False Negatives (FN) & 3572 & 3374 & 2724 & 2598 \\
\hline Precision (global) & 0.73 & 0.95 & 0.97 & 0.98 \\
\hline Sensitivity (global) & 0.51 & 0.54 & 0.62 & 0.72 \\
\hline
\end{tabular}

Table 1. Comparison several people detection methods.

both perform motion segmentation and classification of detected objects. The method $\mathrm{C}$ combines the first two methods for a more robust detection than each one separately. The method from [18] gives the best results and is used as input of the group tracking process. This method learns a background model, resulting in better motion segmentation and better detection of small objects (far from the camera) and static objects. The drawback is the time necessary to learn the model and the low speed of the backgroundsubtraction.

We have performed evaluation of the group tracking algorithm using 4 different views from 3 datasets: videos recorded for the european project VANAHEIM 1 in the Turin subway (figure 6, videos recorded for the european project $\mathrm{ViCoM} \mathrm{d}^{2}$ at the Eindhoven airport (figure 2 and videos from the benchmarking CAVIAR ${ }^{3}$ dataset: the INRIA entrance and the shopping center corridor. In tables 2 and 3 the following metrics are used. The fragmentation metric computes throughout time how many tracked objects are associated with one reference object (ground truth data). The

1 https: / / www . vanaheim-project.eu/

2 http://www.vicomo.org/

3 http://homepages.inf.ed.ac.uk/rbf/CAVIARDATA1/ 


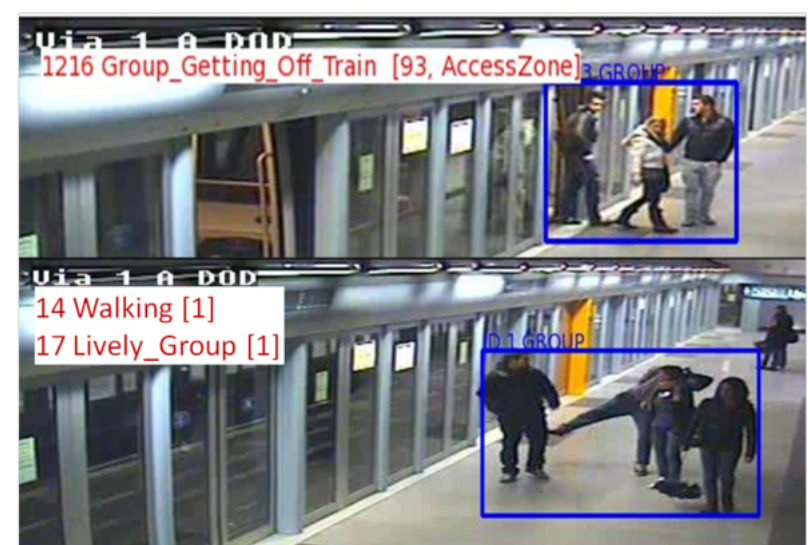

Figure 6. Event detection in Turin subway.

tracking time metric measures the percentage of time during which a reference object is correctly tracked. The purity computes the number of reference object IDs per tracked object. A value close to 1 is significative of a good tracking.

Table 2 shows group detection and tracking results on 16 sequences from the CAVIAR dataset. The first 9 are sequences from INRIA, and the remaining are from the shopping center corridor. One can notice that the shopping view is far more challenging than the hall because more people are visible and there are more occlusions due to the low position of the camera. Table 3 contains the results of this evaluation on 3 annotated sequences (resp. 128, 1373 and 17992 frames) from the Turin subway dataset. In both tables, detection results are good for almost all sequences. In the sequence c2ls1, ground truth groups in the far end of the corridor fail to be detected because of the limitations of the background-subtraction method. Tracking shows good results with a few exceptions. For instance, sequence 2 of table 3 contains a main group present in the foreground for the whole duration of the sequence. This group is correctly tracked with only one id-switch, but many groups are annotated far in the background and are difficult to detect for the motion segmentation algorithm. Their sparse detection results in many id-switches for group tracking. At the best of our knowledge, there is no possibility of comparing our method to an existing one (no public results or code available).

One major achievement of this paper is an ontology for group events based on video sensor (figure 5). The ontology is composed of 49 event models ( 45 models are generic and re-usable in any application with groups (Group_stop, Group_lively,...), 4 models are specifically defined for the applications of this paper (the events depend on the application context, enter shop,...)). The events have been modeled with help of metro surveillance staff.

The results of the group event recognition are given in table 4 for the interesting events. Examples of event recognition are shown in figures 2, 6 and 7 There is only a few

\begin{tabular}{|c|c|c|c|c|c|c|c|c|}
\hline & \multicolumn{5}{|c|}{ Detection } & \multicolumn{3}{|c|}{ Tracking } \\
\hline 离 & $\approx$ & 宁 & $Z_{I}$ & שֶ & $\mid \begin{array}{l}\tilde{0} \\
0 \\
\tilde{n}\end{array}$ & 趈 & $E$ & $\frac{2}{3}$ \\
\hline $\mathrm{fc}$ & 125 & 101 & 3 & 0.55 & 0.98 & 1 & 0.97 & 1 \\
\hline fomd & 159 & 0 & 8 & 1 & 0.95 & 1 & 0.95 & 1 \\
\hline fra1 & 139 & 0 & 67 & 1 & 0.67 & 1 & 0.61 & 1 \\
\hline fra2 & 141 & 0 & 55 & 1 & 0.72 & 1 & 0.70 & 1 \\
\hline $\mathrm{mc1}$ & 231 & 0 & 82 & 1 & 0.74 & 1 & 0.72 & 1 \\
\hline ms3g & 145 & 37 & 37 & 0.80 & 0.80 & 1 & 0.61 & 1 \\
\hline mwt1 & 156 & 0 & 89 & 1 & 0.64 & 1 & 0.28 & 1 \\
\hline mwt2 & 336 & 0 & 268 & 1 & 0.56 & 1 & 0.53 & 1 \\
\hline \begin{tabular}{|l|}
$\mathrm{sp}$ \\
\end{tabular} & 165 & 4 & 36 & 1 & 0.82 & 1 & 0.67 & 1 \\
\hline c2es1 & 858 & 652 & 487 & 0.57 & 0.64 & 0.58 & 0.41 & 0.81 \\
\hline c2es3 & 1093 & 550 & 735 & 0.66 & 0.60 & 0.66 & 0.38 & 0.83 \\
\hline c2ls1 & 788 & 1664 & 655 & 0.32 & 0.55 & 0.50 & 0.13 & 1 \\
\hline c3ps1 & 1298 & 1135 & 210 & 0.54 & 0.86 & 1 & 0.68 & 1 \\
\hline cosme & 1119 & 852 & 35 & 0.57 & 0.97 & 0.25 & 0.60 & 1 \\
\hline csa1 & 269 & 163 & 0 & 0.63 & 1 & 1 & 0.96 & 1 \\
\hline cwbs1 & 2224 & 89 & 1090 & 0.96 & 0.67 & 1 & 0.45 & 0.80 \\
\hline
\end{tabular}

\begin{tabular}{|c|c|c|c|c|c|c|c|c|}
\hline & \multicolumn{5}{|c|}{ Detection } & \multicolumn{3}{|c|}{ Tracking } \\
\hline $\begin{array}{l}5 \\
\qquad\end{array}$ & $\hat{\theta}$ & 记 & 杢 & $\begin{array}{l}\mathscr{D} \\
\stackrel{0}{2}\end{array}$ & 岕 & 焉 & $E$ & 离 \\
\hline 1 & 65 & 0 & 6 & 1 & 0.91 & 1 & 0.86 & 1 \\
\hline 2 & 1346 & 69 & 318 & 0.95 & 0.80 & 0.80 & 0.14 & 0.91 \\
\hline 3 & 6977 & 1677 & 4594 & 0.80 & 0.60 & 0.40 & 0.32 & 0.98 \\
\hline
\end{tabular}

Table 3. Results of group detection and tracking on 3 sequences from the Turin subway. (Prec - Precision, Sens - Sensitivity, Frag - Fragmentation, TT - Tracking Time)

\begin{tabular}{|l|c||c|c|c|}
\cline { 2 - 5 } \multicolumn{1}{c|}{} & GT & TP & FP & FN \\
\hline fighting & 2 & 1 & 0 & 1 \\
\hline split up & 3 & 3 & 0 & 0 \\
\hline joining & 3 & 3 & 0 & 0 \\
\hline shop enter & 5 & 5 & 0 & 0 \\
\hline shop exit & 6 & 6 & 1 & 0 \\
\hline browsing & 3 & 3 & 1 & 0 \\
\hline getting off train & 10 & 9 & 8 & 1 \\
\hline
\end{tabular}

Table 4. Group event recognition for the 3 video datasets

instances of each event because we only focus on meaningful group events. The events are correctly recognized with low false positive and false negative rates. Most of the false positive detections for the event getting off train are due to the fact that the door in the foreground is detected as a person when open. The errors can be corrected by adding a new video primitive: door detector. 

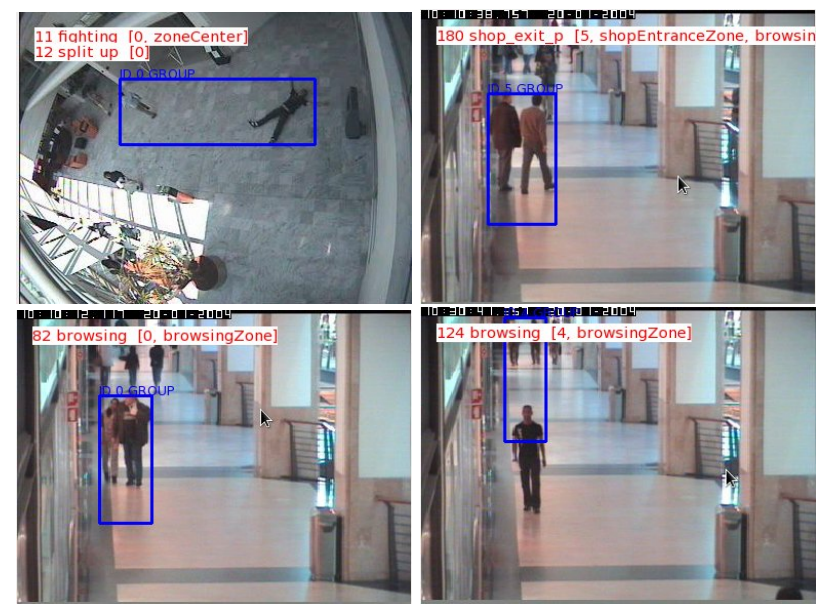

Figure 7. Group event recognition for the CAVIAR sequences. a. Fighting then splitting. b. Exit from the shop. c. Browsing. d. The mis-detected group (ghost due to reflections) is browsing.

\section{Conclusions}

We propose a generic, plug and play framework for event recognition from videos: $S c R e K$. The scientific community can share a common ontology composed of event models and vision primitives. We demonstrate this framework on 4 group behavior recognition applications, using a novel group tracking approach. This approach gives satisfying results even on very challenging datasets (numerous occlusions and long duration sequences) such as in figure 6. The vision primitives are based on global attributes of groups (position, speed, size). The proposed event detection approach correctly recognizes events but shows its limitation for some specific events (e.g. fighting is best characterized by internal group movement). Adapted vision primitives, such as optical flow, solve specific limitations and are easy to plug into $\mathrm{ScReK}$. Moreover, in this work the gap between video data and semantical events is modeled manually by vision experts, the next step is to learn automatically the vision primitives.

Acknowledgment This work was supported partly by the Video-Id, ViCoMo, Vanaheim, and Support projects. However, the views and opinions expressed herein do not necessarily reflect those of the financing institutions.

\section{References}

[1] A. F. Bobick, J. W. Davis, I. C. Society, and I. C. Society. The recognition of human movement using temporal templates. IEEE Trans. on PAMI, 23:257-267, 2001.

[2] O. Brdiczka, J. Maisonnasse, and P. Reignier. Automatic detection of interaction groups. In 2005 International Conference on Multimodal interaction, ICMI '05, Trento It, pages 32-36, 2005.

[3] D. P. Chau, F. Bremond, and M. Thonnat. A multi-feature tracking algorithm enabling adaptation to context variations.
In ICDP, London, Royaume-Uni, Nov. 2011.

[4] X. Chen and C. Zhang. An interactive semantic video mining and retrieval platform-application in transportation surveillance video for incident detection. In ICDM, pages 129-138, 2006.

[5] É. Corvée and F. Bremond. Haar like and LBP based features for face, head and people detection in video sequences. In IWBAVU (ICVS 2011), page 10, Sept. 2011.

[6] T. V. Duong, H. H. Bui, D. Q. Phung, and S. Venkatesh. Activity recognition and abnormality detection with the switching hidden semi-markov model. In $C V P R(1)$, pages 838 845, 2005.

[7] K. Fukunaga and L. Hostetler. The estimation of the gradient of a density function, with applications in pattern recognition. IEEE trans. Information Theory, 21(1):32-40, Jan. 1975.

[8] W. Ge, R. T. Collins, and B. Ruback. Automatically detecting the small group structure of a crowd. In WACV2009, pages 1-8, Dec. 2009.

[9] I. Haritaoglu and M. Flickner. Detection and tracking of shopping groups in stores. In CVPR 2001, volume 1, pages $431-438,2001$

[10] J. Jacques, A. Braun, J. Soldera, S. Musse, and C. Jung. Understanding people motion in video sequences using voronoi diagrams. Pattern Analysis \& Applications, 10:321-332, 2007. 10.1007/s10044-007-0070-1.

[11] G. Lavee, E. Rivlin, and M. Rudzsky. Understanding video events: A survey of methods for automatic interpretation of semantic occurrences in video. IEEE Trans. on Systems, Man, and Cybernetics, Part C, 39(5):489-504, 2009.

[12] W. Lin, M.-T. Sun, R. Poovendran, and Z. Zhang. Group Event Detection With a Varying Number of Group Members for Video Surveillance. IEEE Trans. on Circuits and Systems for Video Technology, 20(8):1057-1067, Aug. 2010.

[13] C. McPhail and R. T. Wohlstein. Using film to analyze pedestrian behavior. Sociological Methods \& Research, 10(3):347-375, 1982.

[14] X. Naturel and J.-M. Odobez. Detecting queues at vending machines: A statistical layered approach. In ICPR2008, pages $1-4$, dec. 2008.

[15] A.-T. Nghiem, F. Bremond, and M. Thonnat. Controlling Background Subtraction Algorithms for Robust Object Detection. In ICICDP2009, Dec. 2009.

[16] R. Romdhane, F. Bremond, and M. Thonnat. A framework dealing with Uncertainty for Complex Event Recognition. In AVSS 2010, Boston, United States, Aug. 2010.

[17] V. T. Vu, F. Bremond, and M. Thonnat. Automatic video interpretation: a novel algorithm for temporal scenario recognition. In IJCAI'03, 2003.

[18] J. Yao and J. Odobez. Multi-layer background subtraction based on color and texture. In CVPR 2007, pages 1-8, 2007. 